\title{
Breit-Wigner Formula for the Scattering Phase in the Stark Effect
}

\author{
Markus Klein ${ }^{1}$, Didier Robert ${ }^{2}$, and Xue-Ping Wang ${ }^{1,3, \star}$ \\ 1 Fachbereich Mathematik MA 7-2, Technische Universität Berlin, D-1000 Berlin 12, FRG \\ 2 Départment de Mathematiques et d'Informatique, Université de Nantes, F-44072 Nantes Cedex, \\ France \\ ${ }^{3}$ Department of Mathematics, Peking University, 100871 Beijing, China
}

\begin{abstract}
Near resonance energy, we study the asymptotic behavior of the derivative of the scattering phase as the applied electric field tends to zero. We obtain the leading asymptotics of the spectral function near a simple resonance, and as an application we rigorously prove the Breit-Wigner formula which relates the width of resonances to the time delay of particles in a homogeneous electric field.
\end{abstract}

\section{Introduction}

Consider the Schrödinger operator of a particle in a weak homogeneous electric field

$$
P(\beta)=P_{0}(\beta)+V(x), \quad P_{0}(\beta)=-\Delta+\beta x_{1},
$$

where $x=\left(x_{1}, x^{\prime}\right) \in \mathbb{R} \times \mathbb{R}^{n-1}$ and $\beta>0$ is a small parameter proportional to the field strength. The resonances of $P(\beta)$ have been studied by several authors $([4,6,8,14,17])$. In particular, in $[14,18]$, upper bounds on the width of resonances were given in the multidimensional case and in $[6,19]$ the asymptotic behavior of the width was analysed. On the other hand, time-delay in scattering theory for the pair $\left(P_{0}(\beta), P(\beta)\right)$ has been studied in $[12,13]$. Many papers of the physics literature affirm an intrinsic relation between the width of resonance and the time-delay. If $V$ is a spherically symmetric potential, it was formally derived in [11] that in a subspace of fixed angular momentum the time-delay near resonance energy is approximately equal to the inverse of the width.

The main purpose of this paper is to rigorously prove this relation for Schrödinger operators with Stark effect in the weak field limit. For the analogous problem in the semiclassical limit we refer to $[5,10]$.

\footnotetext{
* Supported by Alexander von Humboldt Stiftung
} 
Assume that $V$ is a smooth potential satisfying

$$
\left|\partial^{\alpha} V(x)\right| \leqq C_{\alpha}\left\langle x_{1}\right\rangle^{-\sigma_{1}-\alpha_{1}}\left\langle x^{\prime}\right\rangle^{-\sigma_{2}-\left|\alpha^{\prime}\right|}, \quad \forall \alpha=\left(\alpha_{1}, \alpha^{\prime}\right) \in \mathbb{N} \times \mathbb{N}^{n-1}
$$

for some $\sigma_{1}, \sigma_{2}>0$. We remark that our method for obtaining estimates on the spectral function of $P(\beta)$ does require some regularity of $V$, but the smoothness assumption in (1.2) is far from necessary. In fact we need (1.2) just for $|\alpha| \leqq C n$, where $C$ is a universal constant (see Sect. 3). To define resonances, we shall also suppose that $V$ possesses a holomorphic extension in the $x_{1}$-variable into the region

$$
\{z \in \mathbb{C} ; \operatorname{Re} z<-R,|\operatorname{Im} z| \leqq \delta|\operatorname{Re} z|\}
$$

for some $R, \delta>0$ and satisfies there

$$
\left|V\left(x_{1}, x^{\prime}\right)\right| \leqq C\left(\left|\operatorname{Re} x_{1}\right|+\left|x^{\prime}\right|\right)^{-\varepsilon_{0}}, \text { for some } \varepsilon_{0}>0 .
$$

Assuming (1.2) with $\sigma_{1}>\frac{1}{2}$, it was shown in $[12,13]$ that taking the large-space limit of the difference between the sojourn times for $P_{0}(\beta)$ and $P(\beta)$ defines the time-delay operator $T(\beta)$ as a selfadjoint operator in $L^{2}\left(\mathbb{R}^{n}\right)$. In the spectral representation for $P_{0}(\beta), T(\beta)$ is given by a family

$$
T(\lambda, \beta)=-i S(\lambda, \beta)^{*} \frac{d}{d \lambda} S(\lambda, \beta), \quad \lambda \in \mathbb{R},
$$

of operators in $L^{2}\left(\mathbb{R}^{n-1}\right)$, where $S(\lambda, \beta)$ is the scattering matrix for the pair $\left(P_{0}(\beta), P(\beta)\right)$. If $\sigma_{1}>(n+1) / 2, \sigma_{2}>n-1$, then $T(\lambda, \beta)$ is trace class, and we define

$$
\tau(\lambda, \beta)=\operatorname{tr} T(\lambda, \beta) \text {. }
$$

We remark that $\tau(\cdot, \beta)$ is equal to the derivative of the scattering phase for $\left(P_{0}(\beta)\right.$, $P(\beta)$ ). Now let $\lambda_{0}<0$ be an eigenvalue of $P(0)$ with multiplicity $m$.

Assuming (1.2) and (1.3) one knows that there are exactly $m$ resonances, counted with their algebraic multiplicity, of $P(\beta)$ near $\lambda_{0}$, whose widths are all exponentially small as $\beta \downarrow 0([14,18])$. We are concerned with the asymptotic behavior of $\tau(\lambda, \beta)$, as $\beta \rightarrow 0$, for $\lambda$ near the resonance energy. Our main result is

Theorem 1.1. Let (1.2), (1.3) be satisfied with $\sigma_{1}>(n+1) / 2, \sigma_{2}>n-1$. Let $\lambda_{0}<0$ be a simple eigenvalue of $P(0)$ and $z(\beta)$ be the unique resonance of $P(\beta)$ near $\lambda_{0}$ satisfying $z(\beta)=\lambda_{0}+O(\beta)$. Then for $\left|\lambda-\lambda_{0}\right|$ sufficiently small one has

$$
\tau(\lambda, \beta)=2 \operatorname{Im} \frac{1}{z(\beta)-\lambda}+O\left(\beta^{\sigma_{1}-2}\right)+O_{\varepsilon}\left(\frac{e^{-S(\beta)+\varepsilon / \beta}}{|\lambda-z(\beta)|}\right), \quad \text { as } \quad \beta \downarrow 0,
$$

for every $\varepsilon>0$.

Here $S(\beta)$ denotes the distance between any fixed point $x_{0} \in \mathbb{R}^{n}$, independent of $\beta \downarrow 0$, and the sea

$$
\left\{x \in \mathbb{R}^{n} ; V(x)+\beta x_{1}-\lambda_{0}<0, x_{1}<\left(\lambda_{0}+\varepsilon\right) / \beta\right\}
$$

in the Agmon metric $\left(V(x)+\beta x_{1}-\lambda_{0}\right)_{+} d x^{2}$.

We define the scattering phase $s(\lambda, \beta)$ for $\left(P_{0}(\beta), P(\beta)\right)$ by

$$
\operatorname{det} S(\lambda, \beta)=e^{i s(\lambda, \beta)} \text {. }
$$


Then $s^{\prime}(\lambda, \beta)=\tau(\lambda, \beta)$, and from (1.6) one easily derives the asymptotics of increments of $s(\lambda, \beta)$ at the resonance energy $\lambda=\operatorname{Re} z(\beta)$.

Corollary 1.2. Under the assumptions of Theorem 1.1, let $a(\beta)>0$ be a function of $\beta$ such that

$$
a(\beta) \beta^{\sigma_{1}-2} \rightarrow 0, \quad \frac{a(\beta)}{|\operatorname{Im} z(\beta)|} \rightarrow+\infty, \quad \text { and } \quad e^{-S(\beta)+\varepsilon^{\prime} / \beta} \log \left(1+\frac{a(\beta)}{|\operatorname{Im} z(\beta)|}\right) \rightarrow 0
$$

as $\beta \downarrow 0$, for some $\varepsilon^{\prime}>0$.

Define the increments of the scattering phase near $\lambda_{0}$ by

$$
\Delta_{ \pm} s\left(\lambda_{0}, \beta\right)= \pm s(\operatorname{Re} z(\beta) \pm a(\beta), \beta) \mp s(\operatorname{Re} z(\beta), \beta) .
$$

Then one has

$$
\lim _{\beta \downarrow 0} \Delta_{ \pm} s\left(\lambda_{0}, \beta\right)=\pi
$$

\section{Remarks.}

(a) Formula (1.6) shows that near a simple resonance, the trace of time-delay (or equivalently, the derivative of the scattering phase) exhibits a Breit-Wigner resonance form. We remark that following the physics literature the scattering phase is usually defined through the relation $\operatorname{det} S(\lambda, \beta)=\exp (2 i s(\lambda, \beta))(\operatorname{see}[10])$. Compared to that convention, we obtain an additional factor of 2 in (1.6) and the limit in (1.8) is $\pi$ instead of $\pi / 2$.

(b) There exists a variety of choices for the function $a(\beta)$ satisfying (1.7). In fact, assuming (1.2), eigenvalues of $P(\beta)$ are absent ([2]). Thus $\operatorname{Im} z(\beta) \neq 0$. According to the results on asymptotics of widths of resonances $([6,20])$, one has $\operatorname{Im} z(\beta) \sim$ $\beta^{-\sigma} C_{0} e^{-2 S(\beta)}$, for some $\sigma \in \mathbb{R}, C_{0} \neq 0$. Thus it suffices to take $a(\beta)=O\left(\beta^{-\sigma_{1}+2}\right)$.

(c) The relation between time-delay and resonances was studied in [15] for one-dimensional Stark-like Hamiltonians. Our results (1.6), (1.8) are very similar to those in the semiclassical limit obtained in [5], although technical details are rather different.

The proof of Theorem 1.1 is based on the formula

$$
\tau(\lambda, \beta)=-\frac{2 \pi}{\beta} \int_{\mathbb{R}^{n}} \partial_{x_{1}} V(x) \frac{\partial e}{\partial \lambda}(x, x ; \lambda, \beta) d x,
$$

where $(\partial e / \partial \lambda)(\cdot, ; \lambda, \beta)$ is the spectral function of $P(\beta)$. Equation (1.9) was proved in [13] for $\beta=1$. An examination of the dependence on $\beta$ directly gives (1.9). In order to obtain (1.6) through Eq. (1.9), we shall give leading asymptotics as $\beta \downarrow 0$ for the spectral function $\partial e / \partial \lambda$ for $\lambda$ near the resonance energy, which is of interest in itself. In the semiclassical limit, this problem was considered in [4].

The plan of this paper is as follows: In Sect. 2 we construct a representation formula for the resolvent $R(z, \beta)=(P(\beta)-z)^{-1}$ and establish the estimates on the boundary values of the resolvent near resonance energy: for any $s>\frac{1}{2}$, one has

$$
\left\|\left\langle D_{1}\right\rangle^{-s} R(\lambda \pm i o, \beta)\left\langle D_{1}\right\rangle^{-s}\right\| \leqq C\left(\beta^{-1}+\frac{1}{|\lambda-z(\beta)|}\right)
$$


for $\left|\lambda-\lambda_{0}\right|$ sufficiently small. In Sect. 3 we give the asymptotics of the spectral function $\partial e / \partial \lambda$, and in Sect. 4 we prove Theorem 1.1 and Corollary 1.2.

\section{Resolvent Estimates near Resonance Energy}

Let $R(z, \beta)=(P(\beta)-z)^{-1}, \operatorname{Im} z \neq 0$. It is proved in [20] that

$$
\left\|\left\langle D_{1}\right\rangle^{-s} R(\lambda \pm i o, \beta)\left\langle D_{1}\right\rangle^{-s}\right\| \leqq C \beta^{-1}
$$

for $s>\frac{1}{2}$ and $\beta>0$ sufficiently small, locally uniformly in $\lambda \in \mathbb{R} \backslash \sigma(P(0))$. Here $D_{1}=-i \partial_{x_{1}}$. When $\lambda_{0} \in \sigma_{p p}(P(0))$, there exist resonances of $P(\beta)$ near $\lambda_{0}$ and one cannot hope (2.1) to be true for $\lambda$ in a neighborhood of $\lambda_{0}$. To estimate $R(\lambda \pm i o, \beta)$ in this case, we shall first construct a representation formula for $R(\lambda \pm i o, \beta)$ by Grushin's method ([7]), which allows to isolate the contribution of resonances. To be precise, let us recall the definition of resonances in Stark effect by the method of analytic distortion $([9,14,18])$. For $\eta>0$ sufficiently small, we take $\chi_{0} \in C^{\infty}(\mathbb{R})$ such that $\chi_{0}(t)=1$ if $t<\eta / 2$ and $\chi_{0}(t)=0$ if $t>\eta$. For $\theta \in \mathbb{R}$ we set

$$
\varphi\left(x_{1}, \theta\right)=e^{\theta \chi_{0}\left(\beta\left(x_{1}-\lambda_{0} / \beta\right)\right)}\left(x_{1}-\lambda_{0} / \beta\right)+\lambda_{0} / \beta .
$$

Then, for $|\theta|$ sufficiently small, $U(\theta)$ defined by

$$
U(\theta) f(x)=\left(\partial_{x_{1}} \varphi\left(x_{1}, \theta\right)\right)^{1 / 2} f\left(\varphi\left(x_{1}, \theta\right), x^{\prime}\right), \quad f \in L^{2}\left(\mathbb{R}^{n}\right),
$$

is unitary on $L^{2}\left(\mathbb{R}^{n}\right)$. By assumption $(1.3), P(\beta, \theta)=U(\theta) P(\beta) U(\theta)^{-1}$ has a natural holomorphic extension in $\theta$ into a small complex neighborhood of 0 . The resonances of $P(\beta, \theta)$ near $\lambda_{0}$ are defined to be the eigenvalues of $P(\beta, \theta)$ with $\operatorname{Im} \theta>0$ near $\lambda_{0}$. They are essentially independent of $\theta$ and of the choice of $\chi_{0}([18])$. If $\lambda_{0}<0$ is an eigenvalue of $P(0)$ with multiplicity $m$, then it is known $([14,18])$ that there exist exactly $m$ resonances $z_{1}(\beta), \ldots, z_{m}(\beta)$ near $\lambda_{0}$ satisfying

$$
z_{j}(\beta)=\lambda_{0}+O(\beta), \quad \operatorname{Im} z_{j}(\beta)=\tilde{O}\left(e^{-2 S(\beta)}\right) .
$$

Here $S(\beta)$ is the Agmon distance defined in Theorem 1.1 and the notation $\tilde{O}(\cdot)$ is that of Helffer-Sjöstrand [7]. In our context it means that for any $\varepsilon>0$ we can choose the parameter $\eta>0$ so small that

$$
\left|\tilde{O}\left(e^{-2 S(\beta)}\right)\right| \leqq C_{\varepsilon} e^{-2 S(\beta)+\varepsilon / \beta} \text {, for } \beta>0 \text { sufficiently small. }
$$

For earlier results on the stability of resonances, see [8] and the references cited therein.

Henceforth $\theta \in \mathbb{C}$ with $|\theta|$ small and $\operatorname{Im} \theta>0$ is fixed. We also assume that the resonances of $P(\beta)$ near $\lambda_{0}$ correspond to simple poles of the meromorphic extension of the resolvent $R(z, \beta)$. (In the multiple pole case, one would expect a more complicated resonance form for the scattering phase than the Breit-Wigner form [11]. In the semiclassical case, one knows that multiple poles really occur [16]). Under the above assumptions, we can find $m$ functions $u_{j}$ in $L^{2}\left(\mathbb{R}^{n}\right)$ such that

$$
P(\beta, \theta) u_{j}=z_{j}(\beta) u_{j}, \quad\left\|u_{j}\right\|=1 .
$$

For $z$ near $\lambda_{0}$ we consider the Grushin problem

$$
\mathscr{P}_{0}(z)=\left(\begin{array}{cc}
P(\beta, \theta)-z & R_{0}^{-}(\theta) \\
R_{0}^{+}(\theta) & 0
\end{array}\right): \mathscr{D} \times \mathbb{C}^{m} \rightarrow L^{2} \times \mathbb{C}^{m},
$$


where $\mathscr{D}$ denotes the domain of $P(\beta, \theta)$ in $L^{2}\left(\mathbb{R}^{n}\right)$, and $R_{0}^{-}(\theta): \mathbb{C}^{m} \rightarrow L^{2}\left(\mathbb{R}^{n}\right)$, $R_{0}^{+}(\theta): L^{2}\left(\mathbb{R}^{n}\right) \rightarrow \mathbb{C}^{m}$ are defined by

$$
R_{0}^{-}(\theta) c=\sum_{j=1}^{m} c_{j} u_{j} \quad \forall c=\left(c_{1}, \ldots, c_{m}\right) \in \mathbb{C}^{m}
$$

and

$$
\left.R_{0}^{+}(\theta) u=\left(\left\langle u, u_{1}^{*}\right\rangle, \ldots,\left\langle u, u_{m}^{*}\right\rangle\right) \quad \forall u \in L^{2}\left(\mathbb{R}^{n}\right)\right),
$$

where $u_{j}^{*}$ is the eigenfunction of $P(\beta, \theta)^{*}$ such that

$$
P(\beta, \theta)^{*} u_{j}^{*}=\overline{z_{j}(\beta)} u_{j}^{*} ; \quad\left\langle u_{j}, u_{k}^{*}\right\rangle=\delta_{j k} \quad \text { for } \quad j, k=1,2, \ldots, m .
$$

We define a projection $\Pi$ from $L^{2}\left(\mathbb{R}^{n}\right)$ onto the space generated by $u_{1}, \ldots, u_{m}$ by $\Pi u=\sum_{j}\left\langle u, u_{j}^{*}\right\rangle u_{j}$ and set

$$
P^{\prime}(\beta, \theta)=(1-\Pi) P(\beta, \theta)(1-\Pi) ; \quad R^{\prime}(z ; \beta, \theta)=\left(P^{\prime}(\beta, \theta)-z\right)^{-1}(1-\Pi), \quad \operatorname{Im} z>0 .
$$

Then $R^{\prime}(z ; \beta, \theta)$ has a holomorphic extension in $z$ into a small complex neighborhood $\Omega$ of $\lambda_{0}$. For $z \in \Omega, \mathscr{P}_{0}(z)$ is invertible and one checks that

$$
\mathscr{P}_{0}(z)^{-1}=\left(\begin{array}{cc}
E_{0}(z, \theta) & E_{0}^{+}(\theta) \\
E_{0}^{-}(\theta) & E_{0}^{+-}(z)
\end{array}\right),
$$

where $E_{0}(z, \theta)=R^{\prime}(z ; \beta, \theta), E_{0}^{ \pm}(\theta)=R_{0}^{\mp}(\theta)$ and $E_{0}^{+-}(z)=\operatorname{diag}\left(z-z_{j}(\beta)\right)$. According to the general theory ([7], Appendix), one has

$$
(P(\beta, \theta)-z)^{-1}=E_{0}(z, \theta)-E_{0}^{+}(\theta) E_{0}^{+-}(z)^{-1} E_{0}^{-}(\theta), \text { for } \quad z \in \Omega \backslash\left\{z_{1}(\beta), \ldots, z_{m}(\beta)\right\} .
$$

We shall now establish a similar representation formula for $(P(\beta)-z)^{-1}$.

Lemma 2.1. Let $\rho \in C^{\infty}\left(\mathbb{R}^{n}, \mathbb{R}\right)$ such that $\rho(x)=0$ for $|x| \leqq 1$ and $\rho(x)=1$ for $|x| \geqq 2$, and set $\widetilde{P}(\beta)=-\Delta+\beta x_{1}+\rho(x / R) V(x), \tilde{R}(z, \beta)=(\widetilde{P}(\beta)-z)^{-1}$. Then, choosing $R>0$ sufficiently large, we have

$$
\left\|\left\langle D_{1}\right\rangle^{-s} \tilde{R}(z, \beta)\left\langle D_{1}\right\rangle^{-s}\right\| \leqq C \beta^{-1}
$$

for any $s>\frac{1}{2}$, and

$$
\left\|\left\langle D_{1}\right\rangle^{-s}\left\langle\beta x_{1}\right\rangle^{-r}\left\langle x_{1}\right\rangle^{-r_{1}} \tilde{R}(z, \beta)\left\langle x_{1}\right\rangle^{-r_{2}}\left\langle\beta x_{1}\right\rangle^{-r}\left\langle D_{1}\right\rangle^{-s}\right\| \leqq C
$$

for $r, r_{1}, r_{2} \geqq 0, s+2 r>\frac{1}{2}$ with $r_{1}+r_{2} \geqq 1$, uniformly in $z \in \Omega \backslash \mathbb{R}$ for some complex neighborhood $\Omega$ of $\lambda_{0}$. Here $\left\langle D_{1}\right\rangle=\left(1+D_{1}^{2}\right)^{1 / 2}$ and $\left\langle x_{1}\right\rangle=\left(1+x_{1}^{2}\right)^{1 / 2}$.

Proof. For $R>0$ sufficiently large, $\tilde{P}(0)$ has no spectrum in a neighborhood of $\Omega$, since $\tilde{P}(0)>\lambda_{0} / 2$. Then it suffices to apply Theorem 2.1 and Corollary 2.6 in [20]. \#

Henceforth $R>0$ will always be fixed according to Lemma 2.1. We shall consider the Grushin problem for $P(\beta)-z, z \in \Omega$,

$$
\mathscr{P}(z)=\left(\begin{array}{cc}
P(\beta)-z & \chi R_{0}^{-}(\theta) \\
R_{0}^{+}(\theta) \chi & 0
\end{array}\right): \mathscr{D} \times \mathbb{C}^{m} \rightarrow L^{2}\left(\mathbb{R}^{n}\right) \times \mathbb{C}^{m} .
$$

Here $\chi \in C^{\infty}\left(\mathbb{R}_{x_{1}}\right)$ is chosen such that $\chi\left(x_{1}\right)=0$ if $x_{1}<\left(\lambda_{0}+\frac{3}{2} \eta\right) / \beta$ and $\chi\left(x_{1}\right)=1$ if 
$x_{1}>\left(\lambda_{0}+2 \eta\right) / \beta$. Take $\psi \in C^{\infty}\left(\mathbb{R}_{x_{1}}\right)$ such that

$$
\psi\left(x_{1}\right)=0 \quad \text { if } \quad x_{1}<\left(c_{0}-\frac{\eta}{2}\right) / \beta \quad \text { and } \quad \psi\left(x_{1}\right)=1 \quad \text { if } \quad x_{1}>\left(c_{0}+\frac{\eta}{2}\right) / \beta
$$

where $c_{0}<0$ is chosen such that

$$
d_{\beta}(\operatorname{supp}(1-\chi), \operatorname{supp} \psi)=S(\beta) / 2+O(\eta / \beta), \quad \beta \downarrow 0,
$$

where $d_{\beta}(\cdot, \cdot)$ denotes the distance in the Agmon metric $\left(V(x)+\beta x_{1}-\lambda_{0}\right)_{+} d x^{2}$, and $\chi$ and $\psi$ are considered as functions of $x=\left(x_{1}, x^{\prime}\right)$.

For $z \in \Omega_{+}=\Omega \cap\{\operatorname{Im} z>0\}$ we define the approximate inverse

$$
\mathscr{F}(z)=\left(\begin{array}{cc}
\chi E_{0}(z, \theta) \psi+\tilde{R}(z, \beta)(1-\psi) & \chi E_{0}^{+}(\theta) \\
E_{0}^{-}(\theta) \psi & E_{0}^{+-}(z)
\end{array}\right) .
$$

A straightforward calculation gives

$$
\mathscr{P}(z) \mathscr{F}(z)=1+\mathscr{K}(z), \quad \text { where } \quad \mathscr{K}(z)=\left(\begin{array}{ll}
K_{11} & K_{12} \\
K_{21} & K_{22}
\end{array}\right)
$$

with

$$
\begin{aligned}
& K_{11}=-W \tilde{R}(z, \beta)(1-\psi)+\left[D_{1}^{2}, \chi\right] E_{0}(z, \theta) \psi, \quad W=(1-\rho) V, \\
& K_{12}=\left[D_{1}^{2}, \chi\right] E_{0}^{+}(\theta), \\
& K_{21}=R_{0}^{+}(\theta)\left(\chi^{2}-1\right) E_{0}(z, \theta) \psi+R_{0}^{+}(\theta) \chi \tilde{R}(z, \beta)(1-\psi), \\
& K_{22}=R_{0}^{+}(\theta)\left(\chi^{2}-1\right) E_{0}^{+}(\theta) .
\end{aligned}
$$

For $s, r \in \mathbb{R}$, we introduce a class of weighted spaces by

$$
H^{s, r}\left(\mathbb{R}^{n}\right)=\left\{f \in \mathscr{S}^{\prime}\left(\mathbb{R}^{n}\right) ;\langle D\rangle^{s}\left\langle\beta x_{1}\right\rangle^{r} f \in L^{2}\right\},
$$

where $\langle D\rangle=(1-\Delta)^{1 / 2} . H^{s, r}$ is equipped with the natural norm $\left\|\langle D\rangle^{s}\left\langle\beta x_{1}\right\rangle^{r} f\right\|_{L^{2}}$. The following result shows the smoothing effect of $\mathscr{K}(z)$.

Proposition 2.2. Let $r \geqq 0, s \in \mathbb{R}$ with $s+2 r>\frac{1}{2}$. Then for any $N \geqq 0, \mathscr{K}(z)$ is $a$ bounded operator from $H^{s, r} \times \mathbb{C}^{m}$ to $H^{s+N, r+N} \times \mathbb{C}^{m}$ satisfying

$$
\|\mathscr{K}(z)\|_{\mathscr{L}\left(H^{s, r} \times \mathbb{C}^{m}, H^{s+N, r+N} \times \mathbb{C}^{m}\right)}=\tilde{O}\left(e^{-S(\beta) / 2}\right), \quad \beta \downarrow 0,
$$

uniformly in $z \in \Omega_{+}$. More precisely one has in the appropriate spaces

$$
\begin{array}{lll}
K_{11}=\tilde{O}\left(e^{-S(\beta) / 2}\right), & K_{12}=\tilde{O}\left(e^{-S(\beta)}\right), \\
K_{21}=\tilde{O}\left(e^{-S(\beta) / 2}\right), & K_{22}=\tilde{O}\left(e^{-2 S(\beta)}\right), & \beta \downarrow 0,
\end{array}
$$

uniformly in $z \in \Omega_{+}$, for some complex neighborhood $\Omega$ of $\lambda_{0}$.

Proof. The proof is similar to those given in [7] and [18], except that we have to work in weighted Sobolev spaces to obtain the uniformity in $z$ up to the real axis. We shall only estimate $K_{11}$. First consider the case $r=0, N=0$.

For an estimate on $\left[D_{1}^{2}, \chi\right] E_{0}(z, \theta) \psi$ we refer to [18]. Thus it remains to treat $W \tilde{R}(z, \beta)(1-\psi)$. Letting

$$
M=\left\{x \in \mathbb{R}^{n} ; x_{1}>\left(\lambda_{0}+\eta\right) / \beta\right\},
$$


we denote by $\widetilde{P}_{D}(\beta)$ the Dirichlet realization of $\widetilde{P}(\beta)$ in M. Shrinking $\Omega$ if necessary, we can show by the method of [18], Sect. 4, that $\widetilde{P}_{D}(\beta)$ has no spectrum in $\Omega$ for $\beta>0$ sufficiently small.

Letting $\chi_{1} \in C^{\infty}$ with bounded derivatives and supp $\chi_{1} \subset M$, one easily derives, using a priori energy estimates, that

$$
\left\|W\left(\tilde{P}_{D}(\beta)-z\right)^{-1} \chi_{1}(1-\psi)\right\|_{\mathscr{L}\left(H^{-s, 0} ; H^{s, 0}\right)}=\tilde{O}\left(e^{-S(\beta) / 2}\right),
$$

for any $s>0$, uniformly in $z \in \Omega$.

In fact, to obtain this estimate for $s=0$ it suffices to note that the distance between supp $W$ and $\operatorname{supp}(1-\psi)$ in the Agmon metric $d_{\beta}$ is $S(\beta) / 2+O(\eta / \beta)$ and then to use the well known identity

$$
\operatorname{Re} \int_{M}\left(\left|\nabla\left(e^{\phi} u\right)\right|^{2}+\left(V+\beta x_{1}-z-|\nabla \varphi|^{2}\right) e^{2 \phi}|u|^{2}\right) d x=\operatorname{Re} \int_{M} e^{2 \phi}\left(\tilde{P}_{D}(\beta)-z\right) u \cdot \bar{u} d x
$$

for $u=\left(\tilde{P}_{D}-z\right)^{-1} W g$ with $g \in L^{2}\left(\mathbb{R}^{n}\right)$ and $\phi=(1-\varepsilon) d_{\beta}\left(x_{0}, \cdot\right)$ with $\varepsilon>0$. To prove (2.10) in general, one then estimates

$$
(1-\Delta)^{N} W\left(\tilde{P}_{D}-z\right)^{-1} \chi_{1}(1-\psi)(1-\Delta)^{N}
$$

by successively commuting $(1-\Delta) W$ and $\chi_{1}(1-\psi)(1-\Delta)$ with $\left(\tilde{P}_{D}-z\right)^{-1}$, using (2.10) for $s=0$ and the obvious bound

$$
\left\|\langle D\rangle^{r_{0}} W\left(\tilde{P}_{D}-z\right)^{-1} \chi_{1}\langle D\rangle^{r_{1}}\right\| \leqq C, \text { for } r_{0}+r_{1}=2 .
$$

Next we use the identity

$$
W \tilde{R}(z, \beta)(1-\psi)=W\left(\tilde{P}_{D}(\beta)-z\right)^{-1} \chi(1-\psi)+W\left(\tilde{P}_{D}(\beta)-z\right)^{-1}\left[D_{1}^{2}, \chi\right] \tilde{R}(z, \beta)(1-\psi)
$$

for $\operatorname{Im} z>0$ to derive from Lemma 2.1 that

$$
\left\|\left\langle D_{1}\right\rangle^{s} K_{11}\left\langle D_{1}\right\rangle^{-s}\right\|_{\mathscr{L}\left(L^{2}\right)}=\tilde{O}\left(e^{-S(\beta) / 2}\right),
$$

for $s>\frac{1}{2}$, uniformly in $z \in \Omega_{+}$. This proves (2.9) for $r=0, N=0$. To change the weight in (2.11), we need the following estimate: For any $\varphi \in C_{0}^{\infty}(\mathbb{R})$ and $s>0$, one has

$$
\left\|\left\langle\beta x_{1}\right\rangle^{-s} \varphi(P(\beta))\langle D\rangle^{2 s}\right\| \leqq C, \text { uniformly in } \beta>0 .
$$

As in [13] and [20], this can be proved by the method of commutators.

To prove (2.9) for $K_{11}$ in the general case, consider the operator

$$
\tilde{K}_{11}=\langle D\rangle^{s+N}\left\langle\beta x_{1}\right\rangle^{r+N} K_{11}\langle D\rangle^{-s}\left\langle\beta x_{1}\right\rangle^{-r},
$$

where $r \geqq 0, s \in \mathbb{R}$ with $s+2 r>\frac{1}{2}$ and $N \in \mathbb{N}$ is arbitrary. Since $\left\langle\beta x_{1}\right\rangle$ is bounded on $\operatorname{supp} W$ and $\operatorname{supp} \chi^{\prime}$, we have

$$
\begin{aligned}
\left\|\tilde{K}_{11}\right\| \leqq & C\left\|\langle D\rangle^{s+N} \psi_{1} \tilde{R}(z, \beta)(1-\psi)\langle D\rangle^{-s}\left\langle\beta x_{1}\right\rangle^{-r}\right\| \\
& +C\left\|\langle D\rangle^{s+N+1} \chi_{2} E_{0}(z, \theta)\langle D\rangle^{-s}\left\langle\beta x_{1}\right\rangle^{-r}\right\|,
\end{aligned}
$$

where $\psi_{1} \in C_{0}^{\infty}\left(\mathbb{R}^{n}\right)$ with $\psi_{1}=1$ on supp $W$ and $\chi_{2}$ has a similar support property as $\chi^{\prime}$ and satisfies $\chi_{2}=1$ in a neighborhood of supp $\chi^{\prime}$. Since the second term in (2.13) can be treated more easily, we shall only estimate the first. Clearly it suffices 
to prove

$$
\left\|\langle D\rangle^{N} \psi_{1} \tilde{R}(z, \beta)(1-\psi)\langle D\rangle^{k}\left\langle\beta x_{1}\right\rangle^{-r}\right\|=\tilde{O}\left(e^{-S(\beta) / 2}\right), \quad \beta \downarrow 0,
$$

for any $N \in \mathbb{N}, k \in \mathbb{N},-k+2 r>\frac{1}{2}$, uniformly in $z \in \Omega_{+}$. The case $k, N=0$ follows from (2.11) and (2.12). We shall prove (2.14) by induction on $k$ and $N$. Note that

$$
\langle D\rangle^{N} \psi_{1}=\langle D\rangle^{N-2}\left(\psi_{1}(\tilde{P}(\beta)-z)-\psi_{1}\left(\beta x_{1}+\rho\left(\frac{x}{R}\right) V+1-z\right)+\left[\psi_{1}, D^{2}\right]\right)
$$

and $\psi_{1}(1-\psi)=0$. It follows that

$$
\begin{aligned}
& \left\|\langle D\rangle^{N} \psi_{1} \tilde{R}(z, \beta)(1-\psi)\langle D\rangle^{k}\left\langle\beta x_{1}\right\rangle^{-r}\right\| \\
& \quad \leqq C \sum_{j=1}^{2}\left\|\langle D\rangle^{N-j} \varphi_{j} \tilde{R}(z, \beta)(1-\psi)\langle D\rangle^{k}\left\langle\beta x_{1}\right\rangle^{-r}\right\|,
\end{aligned}
$$

where $\varphi_{j}$ is some smooth function with $\operatorname{supp} \varphi_{j} \subset \operatorname{supp} \psi_{1}, j=1,2$. Similarly from the equation

$$
\begin{aligned}
& \varphi_{j} \tilde{R}(z, \beta)(1-\psi)\langle D\rangle^{k} \\
& \quad=\varphi_{j} \tilde{R}(z, \beta)\left\{\left[D^{2}, \psi\right]+(1-\psi)\left(\rho\left(\frac{x}{R}\right) V+\beta x_{1}+1-z\right)\right\}\langle D\rangle^{k-2},
\end{aligned}
$$

we obtain

$$
\begin{aligned}
& \left\|\langle D\rangle^{N-j} \varphi_{j} \tilde{R}(z, \beta)(1-\psi)\langle D\rangle^{k}\left\langle\beta x_{1}\right\rangle^{-r}\right\| \\
& \quad \leqq C \sum_{\ell=1}^{2}\left\|\langle D\rangle^{N-j} \varphi_{j} \tilde{R}(z, \beta) \tilde{\psi}_{\ell}\langle D\rangle^{k-\ell}\left\langle\beta x_{1}\right\rangle^{-r-1+\ell}\right\|,
\end{aligned}
$$

where $\tilde{\psi}_{\ell}$ in some smooth function with a similar support property as $1-\psi$. Using (2.15) and (2.16), it readily follows by induction that (2.14) is true for any $N, k \in \mathbb{N}$. This proves the estimate on $K_{11}$. The other estimates in (2.9) can be proved in a similar way, using exponential decay estimates for $u_{j}$ (see [18]) and a similar estimate for $u_{j}^{*}$. \#

Proposition 2.3. Let $\mathscr{F}(z)$ be defined by (2.7) Then for any $s \in \mathbb{R}, r \geqq 0$ with $2 r \pm s>\frac{1}{2}$ one has

$$
\|\mathscr{F}(z)\|_{\mathscr{L}\left(H^{s, r} \times \mathbb{C}^{m} ; H^{s,-r} \times \mathbb{C}^{m}\right)}=O\left(\beta^{-1}\right), \quad \beta \downarrow 0
$$

uniformly in $z \in \Omega_{+}$.

Proof. Again we only estimate the term $\tilde{R}(z, \beta)(1-\psi)$. The other terms can be treated more easily. It suffices to show

$$
\left\|\left\langle\beta x_{1}\right\rangle^{-r}\langle D\rangle^{s} \tilde{R}(z, \beta)\langle D\rangle^{-s}\left\langle\beta x_{1}\right\rangle^{-r}\right\| \leqq C \beta^{-1}
$$

for $2 r>|s|+\frac{1}{2}$, uniformly in $z \in \Omega \backslash \mathbb{R}$. Let $\varphi \in C_{0}^{\infty}(\mathbb{R}), \varphi=1$ in a neighborhood of $\boldsymbol{\Omega} \cap \mathbb{R}$. For $z \in \Omega$, we have

$$
\left\|\langle D\rangle^{s}(1-\varphi)(\tilde{P}(\beta)) \tilde{R}(z, \beta)\langle D\rangle^{-s}\right\| \leqq C
$$


uniformly in $\beta$. Letting $\frac{1}{2}<s^{\prime}<2 r-|s|$, we obtain from (2.12)

$$
\begin{gathered}
\left\|\left\langle\beta x_{1}\right\rangle^{-r}\langle D\rangle^{s} \varphi(\tilde{P}(\beta)) \tilde{R}(z, \beta)\langle D\rangle^{-s}\left\langle\beta x_{1}\right\rangle^{-r}\right\| \\
\leqq C\left\|\langle D\rangle^{-s^{\prime}} \tilde{R}(z, \beta)\langle D\rangle^{-s^{\prime}}\right\| \leqq C^{\prime} \beta^{-1},
\end{gathered}
$$

uniformly in $z \in \Omega_{+}$. This proves (2.18). \#

From Proposition 2.2 and 2.3 we see that for $r \geqq 0, s \in \mathbb{R}$ with $2 r-|s|>\frac{1}{2}$, the operator

$$
\mathscr{E}(z)=\mathscr{F}(z)(1+\mathscr{K}(z))^{-1}: H^{s, r} \times \mathbb{C}^{m} \rightarrow H^{s,-r} \times \mathbb{C}^{m}
$$

is bounded and its norm is of order $O\left(\beta^{-1}\right)$ uniformly in $z \in \Omega_{+} . \mathscr{E}(z)$ is a right inverse for $\mathscr{P}(z)$. Similarly we can construct a left-inverse for $\mathscr{P}(z)$. This proves that choosing $\beta_{0}>0$ sufficiently small, $\mathscr{P}(z), z \in \Omega_{+}$, is invertible for $0<\beta \leqq \beta_{0}$ and $\mathscr{P}(z)^{-1}=\mathscr{E}(z)$.

Corollary 2.4. For $r \geqq 0,2 r-|s|>\frac{1}{2}$, one has

$$
\|\mathscr{E}(z)\|_{\mathscr{L}\left(H^{s, r} \times \mathbb{C}^{m} ; H^{s,-r} \times \mathbb{C}^{m}\right)}=O\left(\beta^{-1}\right),
$$

and for any $r \geqq 0, s \geqq 0, s+2 r>\frac{1}{2}$, one has

$$
\|\mathscr{E}(z)-\mathscr{F}(z)\|_{\mathscr{L}\left(H^{s, r} \times \mathbb{C}^{m} ; H^{-s,-r} \times \mathbb{C}^{m}\right)}=\tilde{O}\left(e^{-S(\beta) / 2}\right),
$$

uniformly in $z \in \Omega_{+}$.

Corollary 2.4 follows directly from Proposition 2.2 and 2.3. Writing

$$
\mathscr{E}(z)=\left(\begin{array}{cc}
E(z) & E^{+}(z) \\
E^{-}(z) & E^{+-}(z)
\end{array}\right)
$$

we have

$$
R(z, \beta)=E(z)-E^{+}(z)\left(E^{+-}(z)\right)^{-1} E^{-}(z), \text { for } z \in \Omega_{+} .
$$

The advantage of this representation formula is that $E(z), E^{ \pm}(z)$ and $E^{+-}(z)$ can in principal be calculated up to any order. According to (2.9),

$$
E^{+-}(z)=\operatorname{diag}\left(z-z_{j}(\beta)\right)+\tilde{O}\left(e^{-2 S(\beta)}\right) \text {. }
$$

To identify the zeros of $\operatorname{det} E^{+-}(z)$ with the resonances of $P(\beta)$, we establish the following result.

Lemma 2.5. Let $A=\left\{(f, c) \in L^{2}\left(\mathbb{R}^{n}\right) \times \mathbb{C}^{m} ; f(x)=e^{-a x^{2}} p(x)\right.$ for some $a>0$ and some polynomial $p(\cdot)\}$. Then there exists a complex neighborhood of $\lambda_{0}$, which we still denote by $\Omega$, such that for any $u_{1}, u_{2} \in A$ the map $\left.z \rightarrow<\mathscr{E}(z) u_{1}, u_{2}\right\rangle$ defined for $z \in \Omega_{+}$ has a holomorphic extension into $\Omega$.

Proof. Denoting the distortion operator by $U(\vartheta)$, we set $\tilde{R}(z ; \beta, \vartheta)=$ $U(\vartheta) \tilde{R}(z, \beta) U(\vartheta)^{-1}$ for $\vartheta \in \mathbb{R}$ with $|\vartheta|$ small. We define a natural extension of $U(\vartheta)$ to $L^{2}\left(\mathbb{R}^{n}\right) \times \mathbb{C}^{m}$ by

$$
W(\vartheta)(f, c)=(U(\vartheta) f, c) \quad \forall(f, c) \in L^{2} \times \mathbb{C}^{m} .
$$

Then $\mathscr{F}(z, \vartheta)=W(\vartheta) \mathscr{F}(z) W(\vartheta)^{-1}$ defined for $\vartheta \in \mathbb{R}$ with $|\vartheta|$ small has a natural 
holomorphic extension in $\vartheta$ into some small complex neighborhood of 0 . In fact

$$
\mathscr{F}(z, \vartheta)=\left(\begin{array}{cc}
\chi E_{0}(z, \theta) \psi+\widetilde{R}(z ; \beta, \vartheta)(1-\psi) & \chi E_{0}^{+}(\theta) \\
E_{0}^{-}(\theta) \psi & E_{0}^{+-}(z)
\end{array}\right), \quad \operatorname{Im} z>0 .
$$

For fixed $\vartheta \in \mathbb{C}$ with $\operatorname{Im} \vartheta>0$, say $\vartheta=\theta$, we can easily show that $\tilde{R}(z ; \beta, \vartheta)$ defined for $\operatorname{Im} z>0$ has a holomorphic extension in $z$ into $\Omega$, because $\tilde{P}(\beta)$ has no resonances near $\lambda_{0}$. This shows that $\mathscr{F}(z, \vartheta)$ has a holomorphic extension in $z$ into $\Omega$. A similar result holds for $\mathscr{K}(z)$. Thus $\mathscr{E}(z, \vartheta)=W(\vartheta) \mathscr{E}(z) W(\vartheta)^{-1}$, first defined for $\vartheta$ real and then extended to $\operatorname{Im} \vartheta>0$, extends holomorphically to $z \in \Omega$. This proves the lemma. \#

From Lemma 2.5 and (2.19) we see that for distortion analytic functions $f, g$

$$
\langle R(z, \beta) f, g\rangle=\langle E(z) f, g\rangle-\left\langle E^{+}(z) E^{+-}(z)^{-1} E^{-}(z) f, g\right\rangle
$$

has a meromorphic extension in $z$ into $\Omega$, with poles at the zeros of $\operatorname{det} E^{+-}(z)$. But we already know that the poles of $R(z, \beta)$ near $\lambda_{0}$ are exactly $\left\{z_{1}(\beta), \ldots, z_{m}(\beta)\right\}$, which by assumption are all simple. We conclude that the zeros of $\operatorname{det} E^{+-}(z)$ are exactly $\left\{z_{1}(\beta), \ldots, z_{m}(\beta)\right\}$ and there is no non-trivial Jordan block in the canonical form of $E^{+-}(z)$.

Theorem 2.6. Under assumptions (1.2) and (1.3) let $\lambda_{0}<0$ be an eigenvalue of $P(0)$ such that the resonances $\left\{z_{j}(\beta)\right\}_{1 \leqq j \leqq m}$ of $P(\beta)$ close to $\lambda_{0}$ are simple for $0<\beta \leqq \beta_{0}$ for some small $\beta_{0}>0$. Then for every $s \geqq 0, r \geqq 0$ with $s+2 r>\frac{1}{2}$ there exists $C>0$ such that

$$
\left\|\left\langle D_{1}\right\rangle^{-s}\left\langle\beta x_{1}\right\rangle^{-r} R(\lambda \pm i 0, \beta)\left\langle\beta x_{1}\right\rangle^{-r}\left\langle D_{1}\right\rangle^{-s}\right\| \leqq C\left(\beta^{-1}+\max _{1 \leqq j \leqq m} \frac{1}{\left|\lambda-z_{j}(\beta)\right|}\right)
$$

for $\lambda$ near $\lambda_{0}$ and $0<\beta \leqq \beta_{0}$.

Proof. Note that $\left\|E^{+-}(z)^{-1}\right\| \leqq C \max _{j}\left|z-z_{j}(\beta)\right|^{-1}$ and that the results of Corollary 2.4 remain true if we replace $\langle D\rangle^{s}$ by $\left\langle D_{1}\right\rangle^{s}$ in the definition of $H^{s, r}$ (cf. Lemma 2.1). (2.19) for $R(\lambda+i 0, \beta)$ follows immediately, and taking the adjoint of $R(\lambda+i 0, \beta)$ one obtains the estimate on $R(\lambda-i 0, \beta)$. \#

\section{Asymptotics of the Spectral Function}

Let $e(\lambda, \beta)$ denote the spectral projection of $P(\beta)$ onto the interval $(-\infty, \lambda]$. By Stone's formula, one has at least formally $\partial e / \partial \lambda=1 / 2 \pi i(R(\lambda+i 0, \beta)-R(\lambda-i 0, \beta))$. We shall derive the leading asymptotics of the spectral function $\partial e / \partial \lambda(x, y ; \lambda, \beta)$ which by definition is the distributional kernel of $\partial e / \partial \lambda$. The starting point of our arguments is the representation formula (2.19). We write

$$
\mathscr{E}(z)=\mathscr{F}(z)\left(1-\mathscr{K}(z)+\mathscr{K}(z)^{2}\right)+G(z),
$$

where

$$
G(z)=-\mathscr{F}(z) \mathscr{K}(z)^{3}(1+\mathscr{K}(z))^{-1}
$$


Lemma 3.1. With the notations of Sect. 2, for any $r>(n+1) / 4, n / 2<s<2 r-\frac{1}{2}$, one has

$$
\|G(z)\|_{\mathscr{L}\left(\boldsymbol{H}^{-s, r} \times \mathbb{C}^{m} ; \boldsymbol{H}^{s,-r} \times \mathbb{C}^{m)}\right.}=\tilde{O}\left(e^{-2 S(\beta)}\right), \quad \beta \downarrow 0,
$$

uniformly in $z \in \Omega_{+}$.

Proof. As in [7] and [18] one checks that for every fixed $z \in \Omega_{+}, \mathscr{K}(z)^{3}=\widetilde{O}\left(e^{-2 S(\beta)}\right)$ in $\mathscr{L}\left(L^{2} \times \mathbb{C}^{m}\right)$. By the arguments already used in the proof of Proposition 2.2, we can prove that the norm of $\mathscr{K}(z)^{3}: H^{-s, r} \times \mathbb{C}^{m} \rightarrow H^{s, r} \times \mathbb{C}^{m}$ is of order $\tilde{O}\left(e^{-2 S(\beta)}\right)$, uniformly in $z \in \Omega_{+}$.

Now (3.2) follows from Proposition (2.2) and (2.3). \#

From now on we assume that $\lambda_{0}<0$ is a simple eigenvalue of $P(0)$. Then

$$
E^{+-}(z)=(z-z(\beta))\left(1+\tilde{O}\left(e^{-2 S(\beta)}\right)\right)
$$

where $z(\beta)$ is the unique resonance of $P(\beta)$ near $\lambda_{0}$. We write

$$
\mathscr{F}(z)\left(1-\mathscr{K}(z)+\mathscr{K}(z)^{2}\right)=\left(\begin{array}{cc}
\tilde{E}(z) & \tilde{E}^{+}(z) \\
\tilde{E}^{-}(z) & \tilde{E}^{+-}(z)
\end{array}\right)
$$

and define $A(z)$ and $B(z)$ by

$$
\begin{aligned}
& A(z)=\chi E_{0}(z, \theta)+\tilde{R}(z, \beta)(1-\psi)-\tilde{E}^{+}(z) \tilde{E}^{-}(z)(z-z(\beta))^{-1} \\
& B(z)=R(z, \beta)-A(z), \quad z \in \Omega_{+} .
\end{aligned}
$$

Proposition 3.2. With the above notation, one has for $r>(n+1) / 4, n / 2<s<2 r-\frac{1}{2}$,

$$
\|B(z)\|_{\mathscr{L}\left(H^{-s, r} ; H^{s,-r}\right)}=\tilde{O}\left(e^{-S(\beta) / 2}\right)+\tilde{O}\left(\frac{e^{-2 S(\beta)}}{|z-z(\beta)|}\right), \quad \beta \downarrow 0,
$$

uniformly in $z \in \Omega_{+}$.

In particular, the distributional kernel $K_{1}(x, y ; z, \beta)$ of $B(z)$ is continuous on $\mathbb{R}_{x}^{n} \times \mathbb{R}_{y}^{n}$ and satisfies

$$
\left|K_{1}(x, y ; z, \beta)\right| \leqq C\left\langle\beta x_{1}\right\rangle^{r}\left\langle\beta y_{1}\right\rangle^{r}\left(\tilde{O}\left(e^{-S(\beta) / 2}\right)+\tilde{O}\left(\frac{e^{-2 S(\beta)}}{|z-z(\beta)|}\right)\right)
$$

for any $r>(n+1) / 4$, uniformly in $z \in \Omega_{+}$.

Proof. Equation (3.5) is a consequence of Corollary 2.4, Lemma 3.1, (2.19) and (3.3). Note that $B(z)^{*}$ also satisfies the estimate (3.5). Thus (3.6) follows from a theorem of Agmon-Kannai on kernels of operators in Sobolev spaces [1]. \#

Making use of the expressions (2.8) for $K_{i j}$, we can obtain a formula for the operators $\widetilde{E}^{ \pm}(z)$. After a calculation using the ideas of the proof of Proposition 2.2 one finds

$$
\begin{aligned}
A(z)= & \chi E_{0}(z, \theta) \psi+\tilde{R}(z, \beta)(1-\psi)-\frac{1}{z-z(\beta)}\left(\chi-\tilde{R}(z, \beta)\left[D_{1}^{2}, \chi\right]\right) \\
& \cdot \Pi\left(1-(P(\beta)-z) \tilde{R}(z, \beta)\left(1-\psi+\left[D_{1}^{2}, \chi\right] E_{0}(z, \theta) \psi\right)\right) \\
& +\tilde{O}\left(e^{-S(\beta) / 2}\right)+\tilde{O}\left(e^{-S(\beta)}|z-z(\beta)|^{-1}\right)
\end{aligned}
$$

for $z \in \Omega_{+}$. 
Here $\Pi=E_{0}^{+}(\theta) E_{0}^{-}(\theta)$ is the spectral projection of $P(\beta, \theta)$ associated with the eigenvalue $z(\beta)$. To eliminate any dependence on $\theta$ in (3.7), we next replace $E_{0}(\theta, \beta)$ and $\Pi$ by the corresponding quantities related to the Dirichlet realization $P_{D}(\beta)$ of $P(\beta)$ in $M=\left\{x \in \mathbb{R}^{n} ; x_{1}>\left(\lambda_{0}+\eta\right) / \beta\right\}$.

Lemma 3.3. $P_{D}(\beta)$ has a unique eigenvalue $\lambda(\beta)$ such that

$$
\lambda(\beta)=\lambda_{0}+O(\beta), \quad \lambda(\beta)-z(\beta)=\tilde{O}\left(e^{-2 S(\beta)}\right) .
$$

Let $u_{D}(\beta)$ denote the normalized eigenfunction satisfying $P_{D}(\beta) u_{D}(\beta)=\lambda(\beta) u_{D}(\beta)$, $\left\|u_{D}(\beta)\right\|=1$. Then one can choose $u(\beta, \theta)$ and $u^{*}(\beta, \theta)$ such that

$$
\begin{aligned}
u(\beta, \theta)-u_{D}(\beta) & =\tilde{O}\left(e^{-S(\beta)-d_{\beta}(x, \partial M)}\right), \\
u(\beta, \theta)^{*}-u_{D}(\beta) & =\widetilde{O}\left(e^{-S(\beta)-d_{\beta}(x, \partial M)}\right), \quad x \in M .
\end{aligned}
$$

Let $\Pi_{D}$ denote the projection onto the subspace generated by $u_{D}(\beta)$, and set $\Pi_{D}^{\prime}=1-\Pi_{D}$. Letting $R_{D}^{\prime}(z, \beta)=\left(P_{D}(\beta) \Pi_{D}^{\prime}-z\right)^{-1} \Pi_{D}^{\prime}$, one has

$$
\chi E_{0}(z, \theta) \psi-\chi R_{D}^{\prime}(z, \beta) \psi=\tilde{O}\left(e^{-S(\beta) / 2}\right), \quad \text { in } \mathscr{L}\left(H^{-s, r} ; H^{s, r}\right),
$$

for any $s \geqq 0, r \geqq 0$, uniformly in $z \in \Omega$.

Proof. Equation (3.8) was proved in [18], Sect. 3. Equation (3.10) follows from the construction of the Grushin problem given in [18] by suitably choosing the cut-off functions. Equation (3.9) can be derived as (3.8). \#

Combining Lemma 3.3 and (3.7) yields

$$
\begin{aligned}
R(z, \beta)= & \chi R_{D}^{\prime}(z, \beta) \psi+\tilde{R}(z, \beta)(1-\psi)-\frac{1}{z-z(\beta)}\left(\chi-\tilde{R}(z, \beta)\left[D_{1}^{2}, \chi\right]\right) \\
& \cdot \Pi_{D} \chi\left(1-(P(\beta)-z) \tilde{R}(z, \beta)\left(1-\psi+\left[D_{1}^{2}, \psi\right] R_{D}^{\prime}(z, \beta) \psi\right)\right) \\
& +\tilde{O}\left(e^{-S(\beta) / 2}\right)+\tilde{O}\left(e^{-2 S(\beta)}|z-z(\beta)|^{-1}\right),
\end{aligned}
$$

where the remainder terms are estimated in $\mathscr{L}\left(H^{-s, r} ; H^{s,-r}\right)$, uniformly in $z \in \Omega_{+}$. Making use of the relation

$$
\begin{aligned}
\Pi_{D} \chi(P(\beta)-z) & =(\lambda(\beta)-z) \Pi_{D} \chi+\Pi_{D}\left[\chi, D_{1}^{2}\right] \\
& =(z(\beta)-z) \Pi_{D} \chi+\Pi_{D}\left[\chi, D_{1}^{2}\right]+\widetilde{O}\left(e^{-2 S(\beta)}\right),
\end{aligned}
$$

one obtains in $\mathscr{L}\left(H^{-s, r} ; H^{s,-r}\right)$, for $r, s \geqq 0$ with $2 r-s>\frac{1}{2}$

$$
\begin{aligned}
R(z, \beta)= & \chi R_{D}^{\prime}(z, \beta) \psi+\tilde{R}(z, \beta)(1-\psi)-\frac{1}{z-z(\beta)} \chi \Pi_{D} \chi \\
& +\frac{1}{z-z(\beta)}\left\{\tilde{R}(z, \beta)\left[D_{1}^{2}, \chi\right] \Pi^{D} \chi+\chi \Pi^{D}\left[\chi, D_{1}^{2}\right] \tilde{R}(z, \beta)\right. \\
& \left.\cdot\left(1-\psi+\left[D_{1}^{2}, \chi\right] R_{D}^{\prime}(z, \beta) \psi\right)\right\}+\tilde{O}\left(e^{-S(\beta) / 2}\right)+\tilde{O}\left(e^{-2 S(\beta)}|z-z(\beta)|^{-1}\right),
\end{aligned}
$$

uniformly in $z \in \Omega_{+}$.

Applying the same arguments to the operator $P(\beta, \theta)$ with $\operatorname{Im} \theta<0$, we see that (3.11) remains true for $z \in \Omega_{-}=\Omega \cap\{\operatorname{Im} z<0\}$, provided we replace $z(\beta)$ by its 
complex conjugate $\overline{z(b)}$. Note that $R_{D}^{\prime}(z, \beta)$ is holomorphic for $z \in \Omega$. We set $f(\beta)=\chi u_{D}(\beta)$ and assume without loss of generality that $\chi$ and $u_{D}(\beta)$ are real. Combining (3.11) for $z \in \Omega_{ \pm}$with Stone's formula, it follows that

$$
\begin{aligned}
\frac{\partial e}{\partial \lambda}= & \frac{\partial \tilde{e}}{\partial \lambda}(1-\psi)-\operatorname{Im} \frac{1}{\pi(\lambda-z(\beta))} f(\beta) \otimes f(\beta)+F(\lambda, \beta)+\tilde{O}\left(e^{-S(\beta) / 2}\right) \\
& +\widetilde{O}\left(e^{-2 S(\beta)}|\lambda-z(\beta)|^{-1}\right)
\end{aligned}
$$

in $\mathscr{L}\left(H^{-s, r} ; H^{s,-r}\right)$, uniformly in $\lambda$ in a neighborhood of $\lambda_{0}$.

Here $\partial e / \partial \lambda$ and $\partial \tilde{e} / \partial \lambda$ denote the derivative with respect to energy of the spectral projections of $P(\beta)$ and $\widetilde{P}(\beta)$ respectively, and $F(\lambda, \beta)$ is obtained from the boundary values of the fourth term in (3.11).

Finally we can state the main result of this section.

Theorem 3.4. Let $\partial e / \partial \lambda(\cdot, \cdot ; \lambda, \beta)$ and $\partial \tilde{e} / \partial \lambda(\cdot, \cdot ; \lambda, \beta)$ denote the spectral function of $P(\beta)$ and $\tilde{P}(\beta)$ respectively. Let $K_{F}(\cdot, ;, \lambda, \beta)$ denote the distributional kernel of $F(\lambda, \beta)$. Then for $r>n+1 / 4$ and for $\lambda$ in a small neighborhood of $\lambda_{0}$, we have

$$
\begin{aligned}
& \frac{\partial e}{\partial \lambda}(x, y ; \lambda, \beta)-\frac{\partial \tilde{e}}{\partial \lambda}(x, y ; \lambda, \beta)\left(1-\psi\left(y_{1}\right)\right)+\operatorname{Im} \frac{1}{\pi(\lambda-z(\beta))} f(x, \beta) f(y, \beta)-K_{F}(x, y ; \lambda, \beta) \\
& \quad=\left\langle\beta x_{1}\right\rangle^{r}\left\langle\beta y_{1}\right\rangle^{r}\left\{\tilde{O}\left(e^{-S(\beta) / 2}\right)+\tilde{O}\left(e^{-2 S(\beta)}|\lambda-z(\beta)|^{-1}\right)\right\}
\end{aligned}
$$

as $\beta \downarrow 0$, uniformly in $x, y \in \mathbb{R}^{n}$.

Theorem 3.4 follows immediately from (3.12) by taking $(n / 2)<s<2 r-\frac{1}{2}$.

We remark that the remainder estimate in (3.13) is not optimal in the sense that it does not show the expected off-diagonal decrease of a spectral function. But our results become better when restricted to the diagonal $x=y$.

The semiclassical analogue of (3.13) was shown by Gerard and Martinez in [4].

\section{Proof of the Main Results}

Lemma 4.1. Let $\rho(\cdot)$ be a smooth positive function on $\mathbb{R}$ such that $\rho\left(x_{1}\right)=1$ for $x_{1}>0$ and $\rho\left(x_{1}\right)=\left\langle x_{1}\right\rangle$ for $x_{1}<-1$. Then for any $\left.k \geqq 0, s\right\rangle(n+1) / 2$, we have:

$$
\left|\frac{\partial \tilde{e}}{\partial \lambda}(x, x ; \lambda, \beta)\right| \leqq C_{k s} \beta^{k}\left\langle x_{1}\right\rangle^{k+1} \rho\left(\beta x_{1}\right)^{s}, \quad x \in \mathbb{R}^{n}
$$

for $\lambda$ in a small neighborhood of $\lambda_{0}$.

Proof. Since $\lambda_{0} \notin \sigma(\tilde{P}(0))$, for $\lambda$ sufficiently near $\lambda_{0}$, one has: $\lambda \notin \sigma(\tilde{P}(0))$. By Corollary 2.6 in [20], one has for any $s>\frac{1}{2}, k \geqq 0, r=(k+1) / 2$,

$$
\left.\left.\left\|\left\langle D_{1}\right\rangle^{-s}\left\langle x_{1}\right\rangle^{-r} \frac{\partial \tilde{E}}{\partial \lambda}\left\langle x_{1}\right\rangle^{-r}\left\langle D_{1}\right\rangle^{-s}\right\| \leqq C_{k s} \beta^{k}, \quad \beta \in\right] 0, \beta_{0}\right] .
$$

Here $\partial \tilde{E} / \partial \lambda=(1 / 2 \pi i)(\tilde{R}(\lambda+i 0, \beta)-\tilde{R}(\lambda-i 0, \beta))$. Note that for any $m \in \mathbb{N}$,

$$
(\tilde{P}(\beta)+i)^{-m} \frac{\partial \tilde{E}}{\partial \lambda}=(\lambda+i)^{-m} \frac{\partial \tilde{E}}{\partial \lambda},
$$


and by successive commutator estimates, one can show that

$$
\left\|\left\langle D_{1}\right\rangle^{2 s}\left\langle x_{1}\right\rangle^{r}(\tilde{P}(\beta)+i)^{-m}\left\langle x_{1}\right\rangle^{-r} \rho\left(\beta x_{1}\right)^{-s}\right\| \leqq C_{s r}, \quad \text { for } \quad 0 \leqq s \leqq m, \quad r \in \mathbb{R},
$$

uniformly in $\beta>0$. Consequently it follows that for $s>(n+1) / 4,(n / 2)<\sigma<2 s-\frac{1}{2}$, $r=(k+1) / 2$,

$$
\left\|\left\langle x_{1}\right\rangle^{-r} \rho\left(\beta x_{1}\right)^{-s} \frac{\partial \tilde{E}}{\partial \lambda} \rho\left(\beta x_{1}\right)^{-s}\left\langle x_{1}\right\rangle^{-r}\right\|_{\mathscr{L}\left(H^{-\sigma, 0} ; H^{\sigma, 0}\right)} \leqq C \beta^{k} .
$$

By Agmon-Kannai's kernel theorem, one derives that

$$
\left|\frac{\partial \tilde{e}}{\partial \lambda}(x, y ; \lambda, \beta)\right| \leqq C^{\prime} \beta^{k} \rho\left(\beta x_{1}\right)^{s} \rho\left(\beta y_{1}\right)^{s}\left\langle x_{1}\right\rangle^{r}\left\langle y_{1}\right\rangle^{r}, \quad x \in \mathbb{R}^{n}, \quad y \in \mathbb{R}^{n} .
$$

This proves (4.1). \#

Proof of Theorem 1.1. Assume (1.2) with $\sigma_{1}>(n+1) / 2, \sigma_{2}>n-1$. Let $(n+1) / 4<$ $r<\left(\sigma_{1} / 2\right)$.

Applying (1.9) and (3.13), we obtain

$$
\begin{aligned}
\tau(\lambda, \beta)= & -\frac{2 \pi}{\beta} \iint_{\mathbf{R}^{n}}\left\{\left(1-\psi\left(x_{1}\right)\right) \frac{\partial \tilde{e}}{\partial \lambda}(x, x ; \lambda, \beta)-\operatorname{Im} \frac{1}{\pi(\lambda-z(\beta))} f(x, \beta)^{2}+K_{F}(x, x ; \lambda, \beta)\right\} \\
& \cdot \partial_{x_{1}} V(x) d x+\tilde{O}\left(e^{-2 S(\beta)}|\lambda-z(\beta)|^{-1}\right) .
\end{aligned}
$$
parts:

To estimate the integral $I \equiv \iint_{\mathbf{R}^{n}}\left\{\left(1-\psi\left(x_{1}\right)\right)(\partial \tilde{e} / \partial \lambda) \partial_{x_{1}} V d x\right.$, we divide it into two
rts:

$$
I=\left(\int_{x_{1}>C / \beta}+\int_{x_{1} \leqq C / \beta}\right)(1-\psi) \frac{\partial \tilde{e}}{\partial \lambda} \partial_{x_{1}} V d x \equiv I_{1}+I_{2},
$$

where $C>0$ is fixed. For the first term, we apply (4.1) with $k=0$. This gives $I_{1}=O\left(\beta^{\sigma_{1}-1}\right)$. For the second term, we apply (4.1) with $k=\sigma_{1}$. Since $\operatorname{supp}(1-\psi) \subset$ $\left\{x_{1}>-C^{\prime} / \beta\right\}$ for some $C^{\prime}>0, \rho\left(\beta x_{1}\right)$ is bounded on supp $(1-\psi)$. So we have also: $I_{2}=O\left(\beta^{\sigma_{1}-1}\right)$.

Just as in the proof of Proposition 2.2 one can show that the norm of $F(\lambda, \beta)$ as an operator from $H^{-s, r}$ to $H^{s,-r}$ is of order $\widetilde{O}\left(e^{-S(\beta)}|\lambda-z(\beta)|^{-1}\right)$ if $s>(n / 2)$, $r>(s / 2)+\frac{1}{4}$. Thus, as in Theorem 3.4, one obtains that for every $r>(n+1) / 2$,

$$
\left|K_{F}(x, x ; \lambda, \beta)\right| \leqq\left\langle\beta x_{1}\right\rangle^{r} \tilde{O}\left(e^{-S(\beta)}|\lambda-z(\beta)|^{-1}\right), \quad \text { uniformly in } x \in \mathbb{R}^{n}
$$

Thus it follows from (4.2) that

$$
\tau(\lambda, \beta)=\frac{2}{\beta} \operatorname{Im} \frac{1}{\lambda-z(\beta)} \int_{\mathbf{R}^{n}} \partial_{x_{1}} V(x) f(x, \beta)^{2} d x+\widetilde{O}\left(\beta^{\sigma_{1}-2}\right)+\tilde{O}\left(e^{-S(\beta)}|\lambda-z(\beta)|^{-1}\right) .
$$

To calculate the leading term in (4.3) we use the relation

$$
\partial_{x_{1}} V=i\left[D_{1}, P(\beta)\right]-\beta 1 \text {. }
$$

Note that $\left\langle\left[D_{1}, P(\beta)\right] f(\beta), f(\beta)\right\rangle=\tilde{O}\left(e^{-2 S(\beta)}\right)$ and $\|f(\beta)\|^{2}=1+\widetilde{O}\left(e^{-2 S(\beta)}\right)$, since 
$u_{D}(\beta)$ is normalized, and $u_{D}(\beta)$ and its derivative are of order $\tilde{O}\left(e^{-2 S(\beta)}\right)$ on the support of $\chi^{\prime}$. Now (1.6) follows immediately from (4.3). \#

Proof of Corollary 1.2. Assuming (1.2) with $\sigma_{1}>(n+1) / 2$, it is known that $\tau(\lambda, \beta)$ is continuously differentiable with respect to $\lambda$. We let $s(\lambda, \beta)$ denote the scattering phase satisfying $s^{\prime}(\lambda, \beta)=\tau(\lambda, \beta)$ and write $z(\beta)=E(\beta)-i \Gamma(\beta)$ with $\Gamma(\beta)>0$. Then

$$
\begin{aligned}
\Delta_{+} S\left(\lambda_{0}, \beta\right)= & \int_{E(\beta)}^{E(\beta)+a(\beta)} \tau(\lambda, \beta) d \lambda \\
= & 2 \int_{E(\beta)}^{E(\beta)+a(\beta)} \frac{\Gamma(\beta)}{(\lambda-E(\beta))^{2}+\Gamma(\beta)^{2}} d \lambda+O\left(a(\beta) \beta^{\sigma_{1}-2}\right) \\
& +O\left(e^{-S(\beta)}\right) \int_{E(\beta)}^{E(\beta)+a(\beta)} \frac{d \lambda}{|\lambda-z(\beta)|} \\
= & 2 \operatorname{arctg} \frac{a(\beta)}{\Gamma(\beta)}+O\left(a(\beta) \beta^{\sigma_{1}-2}\right)+\widetilde{O}\left(e^{-S(\beta)}\right) \log \left(\frac{a(\beta)}{\Gamma(\beta)}+\sqrt{\left.1+\left(\frac{a(\beta)}{\Gamma(\beta)}\right)^{2}\right)} .\right.
\end{aligned}
$$

Now (1.8) for $\Delta_{+} S\left(\lambda_{0}, \beta\right)$ follows from (4.4) by taking the limit $\beta \downarrow 0$. Equation (1.8) for $\Delta_{-} S\left(\lambda_{0}, \beta\right)$ can be proved in the same way. \#

Our results show that for simple resonances the time-delay has a sharp peak near the resonance energy and the scattering phase increases approximately by $2 \pi$. This agrees well with physical intuition ([11]). We remark that both a peak in time-delay and in the scattering cross-section are characteristic of a resonance process. By establishing a representation formula for the cross-section via the spectral function, we can also study the asymptotic behavior of the cross-section for the pair $\left(P_{0}(\beta), P(\beta)\right)$ in the limit $\beta \downarrow 0$ and justify rigorously the appearance of a peak in the cross-section when the energy is near the resonance energy. Since, however, this is similar in spirit to the semiclassical case, we shall not give the details here. We refer to $[3,5]$ for related problems in the semiclassical case.

Acknowledgements. M. K. and X. P. W. would like to thank Ruedi Seiler for useful discussions on subjects related to this paper, and X. P. W. wants to thank him for his hospitality in Berlin.

\section{References}

1. Agmon, S., Kannai, Y.: On the asymptotic behavior of spectral functions and resolvent kernels of elliptic operators. Israël J. Math. 5, 1-30 (1967)

2. Avron, J. E., Herbst, I. W.: Spectral and scattering theory of Schrödinger operators related to the Stark effect. Commun. Math. Phys. 52, 239-254 (1977)

3. Enss, V., Simon, B.: Total cross sections in non-relativistic scattering theory. In: Quantum mechanics in mathematics, chemistry and physics. Gustafson, K. E., Reinhart, P. (eds.) New York: Plenum Press 1981

4. Gerard, C., Martinez, A.: Semiclassical asymptotics for the spectral function of long range Schrödinger Operators. J. Funct. Anal. 84, 226-254 (1989)

5. Gérard, C., Martinez, A., Robert, D.: Breit-Wigner formulas for the scattering phase and the total scattering cross-section in the semiclassical limit. Commun. Math. Phys. 121, 323-336 (1989) 
6. Harrell, E., Simon, B.: The mathematical theory of resonances whose widths are exponentially small. Duke Math. J. 47, 845-902 (1980)

7. Helffer, B., Sjöstrand, J.: Résonances en limite semiclassique. Bull. S.M.F. Mémoire No. 24/25, tome 114 (1986)

8. Herbst, I.W.: Dilaton analytically in constant electric field. Commun. Math. Phys. 64, 279-298 (1979)

9. Hunziker, V.: Distortion analyticity and molecular resonance curves. Ann. Inst. H. Poincaré 45, 339-358 (1986)

10. Nakamura, S.: Scattering theory for the shape resonance model, II-Resonance scattering. Ann. Inst. H. Poincaré, (1989)

11. Newton, R. G.: Scattering theory of waves and particles, Texts and Monographs in Physics. Berlin, Heidelberg, New York: Springer 1982

12. Robert, D., Wang, X. P.: Time-delay and spectral density for Stark Hamiltonians, I. Existence of time-delay operator. Commun. P.D.E. 14, 63-98 (1989)

13. Robert, D., Wang, X. P.: Time-delay and spectral density for Stark Hamiltonians, II. Asymptotics of trace formulae (to appear)

14. Sigal, I. M.: Bounds on resonance states and width of resonances. Adv. Appl. Math. June 1988

15. Sinha, K.: Time-delay and resonances in simple scattering. In: Quantum mechanics in mathematics, chemistry and physics. Gustafson, K. E., Reinhart, P. (eds.) pp. 99-106. New York: Plenum Press 1981

16. Sjöstrand, J.: Semiclassical resonances generated by non-degenerate critical points. In: Pseudodifferential Operators. Lecture Notes in Mathematics vol. 1256. pp. 402-429. Berlin, Heidelberg, New York: Springer 1987

17. Titchmarsh, E. C.: Eigenfunction Expansions Associated with Second Order Differential Equations, II. Oxford University Press 1958

18. Wang, X. P.: Bounds on widths of resonances for Stark Hamiltonians. Acta Math. Sinica, Ser. B (1990)

19. Wang, X. P.: Asymptotics on width of resonances for Stark Hamiltonians, to appear; and also conference on "Topics on Pseudo-differential Operators", Oberwolfach, June 1989

20. Wang, X. P.: Weak coupling asymptotics of Schrödinger operators with Stark effect, to appear. In: Harmonic Analysis. Lecture Notes in Math., Nankai subser., Berlin, Heidelberg, New York: Springer

21. Yajima, K.: Spectral and scattering theory for Schrödinger operators with Stark effect, II. J. Fac. Sci. Univ. Tokyo, 28A, 1-15 (1981)

Communicated by B. Simon

Received October 18, 1989 